\title{
Estimates for projections in Banach spaces and existence of direct complements
}

\author{
by \\ Gunther DirR (Würzburg), Vladimir RAKočEvić (Niš) \\ and HARALD K. Wimmer (Würzburg)
}

\begin{abstract}
Let $W$ and $L$ be complementary subspaces of a Banach space $X$ and let $P(W, L)$ denote the projection on $W$ along $L$. We obtain a sufficient condition for a subspace $M$ of $X$ to be complementary to $W$ and we derive estimates for the norm of $P(W, L)-P(W, M)$.
\end{abstract}

1. Introduction. The starting point of our investigation is the following result which combines Theorem 5.2 of Berkson [1] with a characterization of minimal angles of Gurariu [2, p. 200].

Theorem 1.1. Let $X$ be a Banach space and let $W, L$, and $M$ be closed subspaces of $X$. Assume

$$
X=W \oplus L
$$

and $L \neq 0$. Let $P(W, L)$ be the projection on $W$ along $L$ and let $\theta(L, M)$ denote the gap between $L$ and $M$. If

$$
\max \{\|P(L, W)\|,\|P(W, L)\|\} \theta(L, M)<1
$$

then $M$ is also complementary to $W$, i.e., $X=W \oplus M$, and

$$
\|P(L, W)-P(M, W)\| \leq \frac{\|P(L, W)\| \theta(L, M)}{1-\|P(L, W)\| \theta(L, M)} \cdot\|P(W, L)\| .
$$

In this note we want to prove a result which contains Theorem 1.1 as a special case. We shall obtain a sufficient condition for $M$ to be complementary to $W$ that is weaker than (1.1) and our estimate for $\| P(L, W)$ $P(M, W) \|$ will be sharper than (1.2).

We shall use the following notation. Let $U$ and $V$ be closed nonzero subspaces of $X$ and define

$$
\delta(U, V)=\sup \{\operatorname{dist}(u, V) ; u \in U,\|u\|=1\} .
$$

2000 Mathematics Subject Classification: Primary 46B20.

Key words and phrases: projections in Banach spaces, complementary subspaces, gap metric. 
Then $\theta(U, V)=\max \{\delta(U, V), \delta(V, U)\}$ is the gap between $U$ and $V$ (see e.g. [3, p. 197]). The range of a linear operator $T$ will be denoted by $\mathcal{R}(T)$.

2. Auxiliary results. In this section $X$ is a real or complex Banach space with a direct sum decomposition $X=W \oplus L$, and $W, L \neq 0$, and $M$ are closed subspaces of $X$. For our purposes the map $P(L, W)_{\mid M}: M \rightarrow L$ will be important.

Lemma 2.1. (a) We have

$$
X=W \oplus M
$$

if and only if the map $P(L, W)_{\mid M}: M \rightarrow L$ has an inverse. Suppose

$$
Q=\left(P(L, W)_{\mid M}\right)^{-1}
$$

exists. Then $Q$ is bounded and

$$
Q=P(M, W)_{\mid L}
$$

(b) Assume

$$
\mu:=\left\|P(W, L)_{\mid M}\right\|<1 .
$$

Then the map $P(L, W)_{\mid M}: M \rightarrow L$ is one-to-one and its range is closed.

(c) If $X=W \oplus M$ and $\mu<1$ then

$$
\|Q\|<\frac{1}{1-\mu} .
$$

Proof. (a) Suppose $Q: L \rightarrow M$ satisfies

$$
Q P(L, W)_{\mid M}=I_{M} \quad \text { and } \quad P(L, W)_{\mid M} Q=I_{L} .
$$

By the Open Mapping Theorem, $Q$ is continuous, and

$$
(Q \cdot P(L, W))^{2}=Q \cdot \underbrace{P(L, W) \cdot Q}_{I_{L}} \cdot P(L, W)=Q \cdot P(L, W)
$$

shows that $Q P(L, W)$ is a projection on $M$ along $W$, which proves (2.1) and (2.2). Conversely, if (2.1) holds then $Q=P(M, W)_{\mid L}$ is well defined and satisfies (2.5).

(b) From (2.3) we obtain

$$
\left\|P(L, W)_{\mid M} x\right\|=\left\|x-P(W, L)_{\mid M} x\right\| \geq(1-\mu)\|x\| \quad \text { for all } x \in M .
$$

Hence the restriction $P(L, W)_{\mid M}$ is bounded from below, which implies injectivity and closed range.

(c) We consider $(2.6)$ with $x=Q y, y \in L$. Then $\|y\| \geq(1-\mu)\|Q y\|$, which yields (2.4).

The decomposition $X=W \oplus L$ implies $X^{*}=W^{\perp} \oplus L^{\perp}$. Thus, in the next lemma, the projection $P\left(L^{\perp}, W^{\perp}\right)$ is well defined. 
Lemma 2.2. The map $P\left(L^{\perp}, W^{\perp}\right)_{\mid M^{\perp}}: M^{\perp} \rightarrow L^{\perp}$ is one-to-one if and only if

$$
\overline{\mathcal{R}\left(P(L, W)_{\mid M}\right)}=L .
$$

Proof. Injectivity of the map $P\left(L^{\perp}, W^{\perp}\right)_{\mid M^{\perp}}$ is clearly equivalent to

$$
(M+W)^{\perp}=M^{\perp} \cap W^{\perp}=0 .
$$

On the other hand we have $\mathcal{R}\left(P(L, W)_{\mid M}\right)=(M+W) \cap L$. Therefore $(2.7)$ is equivalent to

$$
\overline{(M+W) \cap L}=L .
$$

Let us show that (2.8) holds if and only if

$$
\overline{M+W}=X .
$$

Suppose (2.8) holds. Consider $x \in X$ with $x=l+w, l \in L, w \in W$. Because of (2.8) we have $l=\lim s_{\nu}, s_{\nu} \in L$, and

$$
s_{\nu}=m_{\nu}+w_{\nu}, \quad m_{\nu} \in M, w_{\nu} \in W .
$$

Set $x_{\nu}=s_{\nu}+w$. Then $x=\lim x_{\nu}$ and $x_{\nu} \in M+W$. Conversely, assume now (2.9). For $l \in L$ this implies $l=\lim s_{\nu}$ with $s_{\nu}$ as in (2.10). We also have $s_{\nu}=l_{\nu}+\widetilde{w}_{\nu}, l_{\nu} \in L, \widetilde{w}_{\nu} \in W$. Hence, by continuity of $P(W, L)$, we have $\lim \widetilde{w}_{\nu}=0$. Therefore $\lim l_{\nu}=l$, and $l_{\nu} \in(M+W) \cap L$. As (2.9) is equivalent to $(M+W)^{\perp}=0$ the proof is complete.

Lemma 2.3. Let $\delta$ be as defined in (1.3). Then

$$
\left\|P(W, L)_{\mid M}\right\| \leq\|P(W, L)\| \delta(M, L)
$$

and

$$
\left\|P\left(W^{\perp}, L^{\perp}\right)_{\mid M^{\perp}}\right\| \leq\|P(L, W)\| \delta(L, M) .
$$

Proof. If $x \in M,\|x\|=1$, and $\varepsilon>0$ then there exists a $y \in L$ such that $\|x-y\|<\delta(M, L)+\varepsilon$. Then $P(W, L)_{\mid M} x=P(W, L)(x-y)$ yields

$$
\left\|P(W, L)_{\mid M} x\right\|<\|P(W, L)\|(\delta(M, L)+\varepsilon),
$$

which implies (2.11). Note that $\left\|P\left(W^{\perp}, L^{\perp}\right)\right\|=\left\|P(L, W)^{*}\right\|=\|P(L, W)\|$ and $\delta\left(M^{\perp}, L^{\perp}\right)=\delta(L, M)$. Hence (2.12) follows from (2.11).

The following example deals with Berkson's condition (1.1) in Theorem 1.1. It shows that the conditions $\mu=\left\|P(W, L)_{\mid M}\right\|<1$ and $\mu^{*}=$ $\left\|P\left(W^{\perp}, L^{\perp}\right)_{\mid M^{\perp}}\right\|<1$ in part (b) of Theorem 3.1 need not imply (1.1). 
Consider the Euclidean space $\mathbb{R}^{4}$. Set

$$
L=\operatorname{Im}\left(\begin{array}{cc}
1 & 0 \\
0 & 1 \\
\hline 0 & 0 \\
0 & 0
\end{array}\right), \quad W=\operatorname{Im}\left(\begin{array}{cc}
1 & 0 \\
0 & 0 \\
\hline 1 & 0 \\
0 & 1
\end{array}\right), \quad M=\operatorname{Im}\left(\begin{array}{cc}
1 & 0 \\
0 & 1 \\
\hline \tau_{1} & 0 \\
0 & \tau_{2}
\end{array}\right)
$$

To compute $\mu, \mu^{*}$, and the quantities appearing in (1.1) we use the set-up of [4]. If $X=\mathbb{R}^{n}$, and

$$
L=\operatorname{Im}\left(\begin{array}{c}
I_{s} \\
0
\end{array}\right), \quad W=\operatorname{Im}\left(\begin{array}{c}
W_{12} \\
I_{n-s}
\end{array}\right)
$$

and

$$
\begin{gathered}
M=\operatorname{Im}\left(\begin{array}{c}
M_{1} \\
M_{21}
\end{array}\right), \quad M_{1}^{T} M_{1}+M_{21}^{T} M_{21}=I_{s}, \\
M^{\perp}=\operatorname{Im}\left(\begin{array}{c}
M_{12} \\
M_{2}
\end{array}\right), \quad M_{12}^{T} M_{12}+M_{2}^{T} M_{2}=I_{n-s},
\end{gathered}
$$

then $\theta(L, M)=\left\|M_{21}\right\|=\left\|M_{12}\right\|$, and

$$
\|P(W, L)\|^{2}=\|P(L, W)\|^{2}=\left\|I+W_{12}^{T} W_{12}\right\|
$$

and

$$
\mu^{2}=\left\|M_{21}^{T}\left(I+W_{12}^{T} W_{12}\right) M_{21}\right\|, \quad \mu^{* 2}=\left\|M_{12}^{T}\left(I+W_{12} W_{12}^{T}\right) M_{12}\right\| .
$$

In $(2.13)$ we have

$$
W_{12}=\left(\begin{array}{cc}
1 & 0 \\
0 & 0
\end{array}\right), \quad M_{21}=-M_{12}=\left(\begin{array}{cc}
\frac{\tau_{1}}{\sqrt{1+\tau_{1}^{2}}} & 0 \\
0 & \frac{\tau_{2}}{\sqrt{1+\tau_{2}^{2}}}
\end{array}\right) .
$$

Hence

$$
\theta(L, M)^{2}=\max \left\{\frac{\tau_{1}^{2}}{1+\tau_{1}^{2}}, \frac{\tau_{2}^{2}}{1+\tau_{2}^{2}}\right\}, \quad\|P(W, L)\|^{2}=2,
$$

and

$$
\mu^{2}=\mu^{* 2}=\max \left\{\frac{2 \tau_{1}^{2}}{1+\tau_{1}^{2}}, \frac{\tau_{2}^{2}}{1+\tau_{2}^{2}}\right\} .
$$

If we choose $\tau_{1}, \tau_{2}$ such that $0<\tau_{1}<1<\tau_{2}$ then

$$
\frac{2 \tau_{1}^{2}}{1+\tau_{1}^{2}}<1<\frac{2 \tau_{2}^{2}}{1+\tau_{2}^{2}}
$$

which implies $\mu=\mu^{*}<1$ and

$\max \{\|P(W, L)\|,\|P(W, L)\|\} \theta(L, M)=\|P(W, L)\| \theta(L, M)=\frac{\sqrt{2} \tau_{2}}{\sqrt{1+\tau_{2}^{2}}}>1$. 


\section{The main result}

Theorem 3.1. Let $W, L$, and $M$ be closed subspaces of a Banach space $X$. Assume $X=W \oplus L$ and $L \neq 0$. Define

$$
\mu=\left\|P(W, L)_{\mid M}\right\| \quad \text { and } \quad \mu^{*}=\left\|P\left(W^{\perp}, L^{\perp}\right)_{\mid M^{\perp}}\right\| .
$$

(a) If $X=W \oplus M$, then $\mu<1$ implies

$$
\|P(W, L)-P(W, M)\| \leq \frac{\mu}{1-\mu}\|P(L, W)\|,
$$

and $\mu^{*}<1$ implies

$$
\|P(L, W)-P(M, W)\| \leq \frac{\mu^{*}}{1-\mu^{*}}\|P(W, L)\| .
$$

(b) If $\mu<1$ and $\mu^{*}<1$, then $X=W \oplus M$.

Proof. (a) Since both $L$ and $M$ are complementary to $W$ we can use $Q$ in (2.2). We have $P(M, W)=Q P(L, W)$ and

$$
\begin{aligned}
P(W, L)-P(W, M) & =P(W, L)-P(W, L) P(W, M)=P(W, L) P(M, W) \\
& =P(W, L)_{\mid M} Q P(L, W),
\end{aligned}
$$

and the estimate (3.1) follows from (2.4). To prove (3.2) observe that $X^{*}=$ $W^{\perp} \oplus L^{\perp}$ and $X^{*}=W^{\perp} \oplus M^{\perp}$ imply an inequality corresponding to (3.1), namely

$$
\left\|P\left(W^{\perp}, L^{\perp}\right)-P\left(W^{\perp}, M^{\perp}\right)\right\| \leq \frac{\mu^{*}}{1-\mu^{*}}\left\|P\left(L^{\perp}, W^{\perp}\right)\right\|,
$$

which is equivalent to (3.2).

(b) If $\mu^{*}<1$ then Lemma 2.1(b) shows that the map $P\left(L^{\perp}, W^{\perp}\right)_{\mid M^{\perp}}$ : $M^{\perp} \rightarrow L^{\perp}$ is one-to-one. According to Lemma 2.2 this is equivalent to $\overline{\mathcal{R}\left(P(L, W)_{\mid M}\right)}=L$. Similarly, $\mu<1$ implies that $P(L, W)_{\mid M}$ is one-to-one and has closed range. Hence we have $\mathcal{R}\left(P(L, W)_{\mid M}\right)=L$, and $P(L, W)_{\mid M}$ : $M \rightarrow L$ is a bijection. Thus Lemma 2.1(a) yields $X=W \oplus M$.

Corollary 3.2. Let $W, L, M$ be as in Theorem 3.1. Assume $\mu<1$ and

$$
\operatorname{dim} M=\operatorname{dim} L<\infty .
$$

Then $M$ and $W$ are complementary subspaces and (3.1) holds.

Proof. According to Lemma 2.1(b) the map $P(L, W)_{\mid M}: M \rightarrow L$ is one-to-one, and it follows from (3.3) that it has an inverse. Thus, again by Lemma 2.1, we obtain $X=W \oplus M$.

We remark that we cannot discard the condition $\mu^{*}<1$ from Theorem 3.1(b). If a subspace $M$ is topologically isomorphic to $L$, but only the condition $\mu<1$ is satisfied, then $M$ need not be complementary to $W$. Consider the following example. Take $X=\ell_{2}, W=\left\langle e_{1}\right\rangle, L=\left\langle e_{1}\right\rangle^{\perp}=$ 
$\left\{\left(x_{n}\right) \in \ell_{2} ; x_{1}=0\right\}$. Choose $M=\left\{\left(x_{n}\right) \in \ell_{2} ; x_{1}=x_{2}=0\right\}$. Then $M \subseteq L$, $P(W, L)_{\mid M}=0$, and $M$ is topologically isomorphic to $L$, but $X \neq W \oplus M$.

The following proof shows that Theorem 1.1 is an immediate consequence of Theorem 3.1.

Proof of Theorem 1.1. Condition (1.1) implies

$$
\|P(W, L)\| \delta(M, L)<1 \quad \text { and } \quad\|P(L, W)\| \delta(L, M)<1 .
$$

Because of Lemma 2.3 the inequalities (3.4) yield $\mu<1$ and $\mu^{*}<1$. Hence by Theorem 3.1 the subspace $M$ is complementary to $W$. Moreover, since the function $f(t)=t(1-t)^{-1}$ is increasing on $[0,1)$ we obtain the estimate (1.2) from (2.12) and (3.2).

Acknowledgements. We are grateful to Dr. D. Ivers for useful comments and suggestions.

\section{References}

[1] E. Berkson, Some metrics on the subspaces of a Banach space, Pacific J. Math. 13 (1963), 7-22.

[2] V. I. Gurariı̌, On openings and inclinations of subspaces of a Banach space, Teor. Funktsii Funktsional. Anal. i Prilozhen. Vyp. 1 (1965), 194-204 (in Russian).

[3] T. Kato, Perturbation Theory for Linear Operators, 2nd ed., Springer, Berlin, 1996.

[4] H. K. Wimmer, Canonical angles of unitary spaces and perturbations of direct complements, Linear Algebra Appl. 287 (1999), 373-379.

Mathematisches Institut

Universität Würzburg

D-97074 Würzburg, Germany

E-mail: dirr@mathematik.uni-wuerzburg.de

wimmer@mathematik.uni-wuerzburg.de
Department of Mathematics

University of Niš 18000 Niš, Yugoslavia

E-mail:vrakoc@bankerinter.net 